\title{
Adolescencia y Comunicación
}

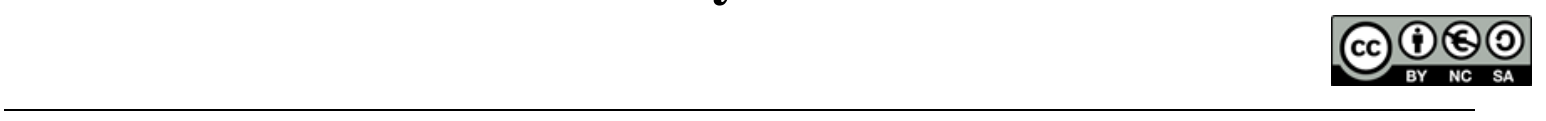

Adolescence and communication

Mariuska Comas Benítez. ${ }^{1}$, Myrian Biviana Pérez Constante. ${ }^{2}$, Byron Enrique Mora Avilés. ${ }^{3}$ \& Milena Aracely Estupiñan Guamani. ${ }^{4}$

Recibido: 05-12-2019 / Revisado: 22-12-2019 /Aceptado: 17-01-2020/ Publicado: 07-02-2020

Abstract.

DOI: $\underline{\text { https://doi.org/10.33262/concienciadigital.v3i1.1.1175 }}$

With the aim of proposing communication games in adolescent students, to optimize the development of group activities in the educational process, the following research was carried out with an eminently qualitative approach in the careers of Basic Education and Initial Education of the Faculty of Human Sciences and of Education. The study had the presence of 3 research teachers who were involved in learning about the changes that occur in adolescents. For this, the methods, synthetic analytical and deductive inductive were used, which allowed to review the existing literature for the description of the games, in turn documentary analysis was also used, which allowed a study of the games most used for group communication. As results, there are three communication games that will be applied throughout the course to the students of the aforementioned careers.

Keywords: Communication, pedagogical process, adolescent, activity, orientation, participatory techniques.

\section{Resumen.}

Con el objetivo de proponer juegos de comunicación en estudiantes adolescentes, para optimizar el desarrollo de las actividades grupales en el proceso educativo, se realizó la siguiente investigación con enfoque eminentemente cualitativo en las carreras de Educación

${ }^{1}$ Universidad Técnica de Ambato. Carrera Educación Básica. Ambato, Ecuador. m.comas@uta.edu.ec

${ }^{2}$ Universidad Técnica de Ambato. Carrera Educación Inicial. Ambato, Ecuador, mb.perez@uta.edu.ec

${ }^{3}$ Universidad Técnica de Ambato Carrera Educación Básica. Ambato, Ecuador, be.mora@uta.edu.ec

${ }^{4}$ Universidad Técnica de Ambato Carrera Educación Básica. Ambato, Ecuador, estupinan@uta.edu.ec 
Básica y Educación Inicial de la Facultad de Ciencias Humanas y de la Educación. El estudio contó con la presencia de 3 docentes investigadores los cuales se involucraron en conocer los cambios que se producen en los adolescentes. Para ello se utilizaron los métodos, analítico sintético e inductivo deductivo, los cuales permitieron revisar la literatura existente para la descripción de los juegas, a su vez también se utilizó el análisis documental que permitió realizar un estudio de los juegos mas utilizados para la comunicación grupal. Se encuentran como resultados, tres juegos de comunicación, que serán aplicado durante todo el curso a los estudiantes de las carreras antes mencionadas.

Palabras claves: Comunicación, Proceso pedagógico, Adolescente, Actividad, orientación, Técnicas participativas.

\section{Introducción.}

Uno de los efectos sociales de la modernización es, sin duda, la transformación del proceso a través del cual los sujetos se vinculan con el grupo al que pertenecen ( Mercado Maldonado \& Hernández Oliva, 2010). En este sentido Es indudable que el tema relacionado con el período de la adolescencia y su comunicación es sugestivo y por las diferencias de criterio, ha suscitado un estudio profundo, sin dejar de reconocer la cantidad de enigmas que presenta. En la actualidad, la creciente necesidad de educar a nuestros hijos en este período y su adecuada comunicación, ha hecho que dicha temática sea introducida en los planes de estudio. Tarea esta que involucra a padres y profesores, en la que cada uno debe asumir de un modo serio esta responsabilidad. (Izquierdo E., 2015)

Para los profesores es muy importante tener en cuenta las formas en que se comunican los adolescentes, con el objetivo de organizar la actividad grupal y el proceso educativo. Es necesario que la comunicación sea asertiva dentro del propio grupo, ya sea oficial o no. Una comunicación bien organizada y con eficiencia educativa influye positivamente en la formación de la personalidad de los adolescentes a partir de la riqueza de la información que se transmite.

Estudios de algunos factores, vías y mecanismos que intervienen en el proceso de formación de la autorregulación moral del comportamiento en la edad juvenil realizado por O.Kraftchenko (1990) citado por (Ojalvo Mitrany, 2002) considera importante el papel de la comunicación para el estudio del desarrollo moral de la personalidad de los adolescentes y jóvenes, al erigirse esta categoría. O.Kraftchenko (1990) En sus investigaciones sobre la 
comunicación pedagógica obtuvo como resultado que los profesores percibidos como modelos son aquellos con los cuales el estudiante se comunica más y mantiene mejores relaciones; por el contrario, no son considerados como tales aquellos con los que el estudiante no se comunica o lo hace poco y las relaciones son peores. Los estudiantes aprecian a los profesores que saben y que son severos, pero justos, benévolos y con tacto, que saben explicar el material de forma interesante y comprensible, organizan rápido el trabajo, atraen a los alumnos y lo hacen productivo para todos y cada uno.

\section{Desarrollo.}

En ocasiones son insuficientes los caminos adecuados para satisfacer las necesidades de los adolescentes y los métodos que se emplean para convencer a los mismos de asumir una u otra conducta, no son los más apropiados. Esto origina contradicciones en diferentes contextos que afecta la formación de la personalidad, el doctor Emilio Ortiz Torres, (1996) las refieres en su tesis citado por (Almeida Macias \& Ortiz Torre, 2016) y modificado por los autores según las características de los estudiantes de primer nivel de la Universidad Técnica de Ambato; por ser los que aún están en la etapa de adolescencia tardía.

En el contexto familiar (Emilio Ortiz Torres, 1996)

1. Existen contradicciones respecto a la independencia.

2. Existen contradicciones respecto al apoyo y a la seguridad.

3. Existen contradicciones respecto a la integración y estabilidad.

4. Existe necesidad de comunicación con temas que sean de su interés.

En el contexto de la escuela_(Emilio Ortiz Torres, 1996)

1. Existen aspiraciones de los padres, las suyas propias y sus capacidades individuales.

2. Existe miedo e inseguridad.

3. No existe motivación por el estudio y la escuela.

4. Existe inseguridad de no ser aceptados por el grupo.

5. Existe esfuerzo por mantener actitudes y conductas que garanticen su integración grupal.

6. Existen relaciones interpersonales tensas y difíciles.

7. Predomina la comunicación con sus coetáneos.

En lo personal (Emilio Ortiz Torres, 1996)

1. Existe inseguridad en la toma de decisiones.

2. Existe búsqueda constante de autoafirmación. 
3. Existe cumplimiento de expectativas.

4. Existen pobres e inadecuados conocimientos de sí mismo e inconformidad con sus características.

5. Existe inseguridad en cuanto a sus posibilidades.

6. Existe inseguridad por falta de orientación.

7. Existe necesidad de un reconocimiento social y en la comunicación con los demás se esfuerzan por lograrlo.

Teniendo en cuenta lo antes expuesto, los adolescentes al comunicarse con los demás tratan de imponer sus criterios (Ahumada, 2010), necesitan que tengan en cuenta sus intereses, motivaciones (Romero Pileta, y otros, 2019), aspiraciones para lograr un reconocimiento social en el grupo, la escuela y la familia. En esta etapa las amistades ganan en valor y el colectivo aparece como una prolongación de la propia familia, a diferencia de la infancia, donde existe una mayor relación de dependencia con la familia y el maestro, ocupando el colectivo un lugar secundario.

La alternativa que se propone se materializa con la aplicación de técnicas participativas y un juego didáctico, que pueden ser muy valiosos para caracterizar a los adolescentes y favorecer su comunicación interpersonal. (Bautista Vallejo \& López , 2002)

Por otra parte, son de una importancia notable el modelo de presencias de estos jóvenes en el espacio de ocio y el grupo de iguales, y su articulación con el espacio familiar. (Barbeta Viñas \& Termes López, 2014) El grupo de iguales se sitúa fuera del instituto, con quienes ponen en práctica actividades de ocio próximas a la experimentación (BONAL, y otros, 2003).

En la concepción del ocio que desarrollan, éste se entiende como espacio de expresión, placer y en alguno de los casos, transgresión. Se observa que este tipo de presencias se vinculan a un control familiar débil del ocio, con un poder de negociación de las normativas familiares por parte de los jóvenes bastante elevado, y con niveles bajos de interiorización de estas normas.

El control escolar por parte de la familia se muestra esporádico o inexistente. Sin embargo, el discurso de estos jóvenes coincide en el tópico común de la construcción de un imaginario futuro con claras influencias del modelo familiar en que se han socializado, otorgando así un papel relevante a los habitus, en su proyección sobre un modelo familiarista tradicional: familia nuclear, hijos, separación de roles, etc (Barbeta Viñas \& Termes López, 2014)

Técnicas participativas que se proponen para caracterizar a los adolescentes.

a. La tarjeta espejo.

b. El árbol caracterizador. 
c. La fruta de la comunicación.

Para aplicar las técnicas participativas es necesario conocer que son disímiles las definiciones dadas a la palabra técnica (García Hernández , Rodríguez Ferra , \& Castaño Hernández, 1999), (Zaldívar, 2004), (Rodríguez Selpa, Socarrás Sánchez, Bujardón Mendoza, \& Iglesias Morel, 2015) pero todos los autores coinciden en que son habilidades para usar los procedimientos, recursos de una ciencia o arte.

Las técnicas recogen hechos situaciones, contenidos, conceptos, valores, reflejan la realidad en sus diversos aspectos, contienen uno o varios elementos que permiten la comunicación en determinados grupos, en forma de códigos estimuladores de análisis de interpretación.

Ese aspecto de la realidad o concepto que se maneja "es traducido" cuando se diseña o aplica una técnica a un determinado tipo de código (visual, audiovisual, auditivo y vivencial) que no es cerrado y por tanto transmisor verticalista, sino "abierto", provocador y generador de un proceso participativo de "decodificación".

Las técnicas que parecen acabadas en su diseño, no son aplicables en forma mecánica, pues su aplicación depende de:

a) Las características del grupo que condicionan el procedimiento de aplicación (pequeño, numeroso, adultos, trabajadores, campesinos, mujeres, mixto, iniciante o con cierta experiencia).

b) Las condiciones objetivas y materiales en que se desarrolla el proceso, no es lo mismo un taller intensivo, que un proceso periódico a largo plazo.

c) Los temas y sobre todo los objetivos que se quieran alcanzar, la particularidad, la intensidad, las características del grupo que condicionan el contenido temático y el fin que se quiera y pueda alcanzar, en este caso las técnicas serán adaptadas a estas condiciones.

d) El eje temático también orienta y condiciona el uso de técnicas y su procedimiento, pues para un mismo tema general pueden escogerse y diseñarse diferentes ejes temáticos que condicionan el enfoque y el análisis del tema y su contenido.

En función de los distintos campos y forma de aplicación de la concepción metodológicodialéctica se puede ubicar el papel y el sentido de las distintas técnicas. Las técnicas pueden ser muy diversas, pero hay que considerarlas solo como instrumentos o herramientas cuya validez principal está en el uso que se les dé y en función de los objetivos para lo que se utilice. Siempre deben incentivar la reflexión, la comunicación y el debate entre los participantes. Se pueden considerar como "armas" en el proceso pedagógico, no como "tácticas" o "estrategias" y para ello es aconsejable: 
- Conocerlas bien, saberlas utilizar y saberlas conducir correctamente.

- Dirigirlas siempre hacia el logro de un objetivo preciso.

- Ponerlas al alcance de los sectores populares para que ellos la puedan utilizar creativamente.

- Conocer y saber usar sus características particulares, sus posibilidades y límites.

- Tener imaginación y creatividad al aplicarlas, y por tanto, modificarlas de acuerdo a las características específicas del grupo y al dinamismo de la reflexión que se logre.

Lo más importante no es escoger la técnica adecuada sino el procedimiento de utilización de la misma. (Zaldívar, 2004) No se trata, por tanto, de usar técnicas novedosas para hacer entretenida y dinámica una actividad educativa, se trata de incentivar una participación ordenada del grupo, que permita arribar a conclusiones claras sobre el tema que se esté tratando, definitivamente se trata de generar un proceso de apropiación de los conocimientos a través de un esfuerzo de interpretación, análisis y síntesis. La concepción dialéctica se expresa también en el procedimiento de aplicación de cada técnica participativa (García Hernández, Rodríguez Ferra , \& Castaño Hernández, 1999)

\section{Orientaciones para desarrollar las técnicas participativas.}

Las técnicas propuestas por los autores y el juego didáctico se estructuran de acuerdo a las indicaciones que aparecen en el libro "Técnicas Participativas de Educadores Cubanos. CIE Graciela Bustillo“.

\section{Metodologia.}

La investigación se enmarca en el ámbito de la Educación, específicamente en el proceso de técnicas participativas. En ella se asume un enfoque cualitativo, para resolver un problema de carácter práctico dentro de la Universidad Técnica de Ambato. Se utilizaron diferentes métodos dentro de los que se encuentran, el analítico sintético, la inducción-deducción, y el análisis documental para lograr dar respuesta a la problemática encontrada.

\section{Población}

El cálculo del número de beneficiarios por alumno (PBA), se realiza contabilizando los estudiantes de octavo semestre de Educación Básica y Educación Inicial de la Universidad Técnica de Ambato, registrados como matricula oficial, los datos se presentan en la siguiente tabla:. 
Tabla 1. Población de la investigación

\begin{tabular}{lcl}
\hline Objeto de estudio & Población & \multicolumn{1}{c}{ Observación } \\
\hline $\begin{array}{l}\text { Octavo de } \\
\text { Educación Básica }\end{array}$ & 34 & $\begin{array}{l}\text { La población representan el total de } \\
\text { las instituciones en las que los } \\
\text { estudiantes de la carrera de Educación } \\
\text { básica marzo - agosto } 2019 . \\
\text { La población representan el total de } \\
\text { las instituciones en las que los } \\
\text { estudiantes de la carrera de Educación } \\
\text { Inicial marzo - agosto 2019. }\end{array}$ \\
Educación Inicial & 42 & $\begin{array}{l}\text { Población } \\
\text { Total }\end{array}$ \\
\hline
\end{tabular}

Fuente: Elaboración propia.

La tabla 1 se muestra los resultados de la población utilizada para la implementación de los juegos.

\section{Resultados}

Tabla 2. Juego didáctico, la tarjeta espejo.

I- Objetivo: Desarrollar el espíritu crítico y autocrítico teniendo en cuenta el tipo y el estilo de comunicación y la cohesión grupal.

II- Materiales: Tarjetas con patrones de conductas positivas y negativas.

III- Desarrollo:

- Sentados en círculo, el educador le da a cada participante un número de orden y una tarjeta.

- Se rotarán las tarjetas y cada participante que considere que tiene la conducta escribirá en la tarjeta su número de orden.

- El educador recoge las tarjetas y de acuerdo con los resultados de este diagnóstico, anima un debate crítico y autocrítico sobre las causas que ocasionen las actitudes negativas .Se puede generar una reflexión colectiva crítica que propicie el cambio, y fortalecer las conductas positivas.

IV- Recomendaciones:

- El educador debe evitar hacer referencias personales, y con mucha ética puede referirse a estos problemas con el propósito de desarrollar la comunicación y fortalecer valores.

Fuente: Elaboración propia. 
Tabla3. Juego didáctico, el árbol caracterizador.

I. Objetivo: Hacer un pequeño árbol de cartulina para cada equipo y uno más grande para todo el grupo.

II. Materiales: Tarjetas con patrones de conductas positivas y negativas.

III. Desarrollo:

- Se divide el grupo en dos equipos, por afinidad de los participantes.

- A cada equipo el educador le entregará dibujado en una cartulina un pequeño árbol y un grupo de frutas, invitándolos a que individualmente coloquen en cada fruto una cualidad positiva que ellos consideren poseen y en otro una cualidad negativa.

- En colectivo se analizarán las cualidades escogidas por cada uno de los integrantes del grupo, y se pueden adicionar otros hasta tanto conformen el árbol del grupo con cualidades positivas y negativas.

- En plenario cada equipo presentará su árbol y se analizan las cualidades positivas, si pueden considerarse cualidades generales del grupo, se coloca una fruta con dicha cualidad en el árbol grande del aula. En cuanto a la cualidad negativa se hace una reflexión crítica, se tratan de analizar las causas y la necesidad de declarar frutas enfermas que deben caer. Las que son generales del grupo se colocan en el árbol grande y si están de acuerdo en erradicarlas, se pinta en la fruta una mancha que hará que con el tiempo y el esfuerzo se desprenda y caiga.

- Cada cierto tiempo una reflexión colectiva de los pequeños grupos permitirá que ellos digan qué nueva fruta adicionar, cuál deben desprender, e igualmente en el árbol grande se irá colocando las que reflejan las conductas positivas de los participantes.

IV. Recomendaciones:

Es útil el empleo de esta técnica con alumnos de primaria y adolescentes con trastornos de conducta.

Fuente: Elaboración propia.

Tabla 4. Juego, la fruta de la comunicación.

I. Objetivos: Diagnosticar, construir o consolidar conocimientos sobre la comunicación.

II. Materiales: Pedazo de papel.

III. Desarrollo:

- El profesor explicará la manera de desarrollar esta nueva competencia para ello dividirá el grupo en equipos y le entregará por escrito un concepto, relacionado con la comunicación, sobre el que se reflexionará. 
- Cada grupo irá escribiendo características del concepto en pedazos de papel con los que irán conformando su fruta preferida (tiempo aproximado: 20 minutos).

- Concluido el tiempo a la voz del profesor los equipos se rotan las frutas y comienzan a descascararlas y debe inferir de qué concepto se trata. Cuando estén convencidos deben decirlo en voz alta y si aciertan será el equipo ganador; si no es el concepto, se considera perdedor y se anula la reflexión; es muy importante recalcar que se dará puntos adicionales a los que digan las vitaminas y minerales que contiene la fruta y su importancia.

- Inferido el concepto, se escriben en papel todas las ideas que cada equipo aportó y a partir de esa reflexión el profesor, en plenaria, establece un diálogo que permita ir construyendo la definición y así profundizar en el tema.

Para la elaboración y el desarrollo del juego didáctico, es importante tener en cuenta las siguientes reflexiones.

- La clase pueden constituir un espacio favorable para la utilización del juego, los profesores deben lograr una buena motivación y orientación en los diferentes momentos de la clase y propiciar la ejecución de las tareas individuales, por parejas, por equipos o por grupos, favoreciendo los procesos de comunicación y socialización que influyen en la adquisición individual del contenido y la formación de hábitos y habilidades.

- El juego, por su naturaleza, dinamiza la clase y adquiere diferentes formas atendiendo a la finalidad por la cual se utiliza y el impacto que se logra con su empleo, en relación con las exigencias de los objetivos de la actividad docente, puede insertarse tanto en la introducción de la clase como en su desarrollo y puede emplearse felizmente, como parte de las conclusiones de la misma.

- El desarrollo de las clases se convierte en un escenario importante para desplegar las acciones lúdicas, para el cumplimiento de sus objetivos específicos y de los objetivos formativos en general. Los juegos, por su naturaleza dinámica y abierta, se corresponden con las funciones didácticas que predominan en las clases, por lo que son muy apropiados para desarrollar el proceso de enseñanza-aprendizaje.

IV. Recomendaciones:

El juego constituye una opción didáctica para realizar conclusiones de las clases, donde prime la creatividad, el intercambio, la comunicación y esto favorece las relaciones interpersonales entre los participantes. Cuando el profesor selecciona los procedimientos lúdicos para esta fase de la clase, propicia que la misma termine de forma agradable, dejando siempre una huella positiva, afectiva en los participantes

Fuente: Elaboración propia. 
Tabla 5. Juego didáctico, aprendiendo a comunicarnos

I. Objetivo: facilitar las reflexiones para profundizar en el contenido referente a la comunicación

II. Materiales: tablero de mesa, tarjetas de cartulina agrupadas en Interrogantes, Hablando de salud, Vale la pena y Entre tú y yo.

III. Desarrollo:

- El profesor primeramente, a partir de una situación importante por la que atraviesa el grupo, prepara un conjunto de tarjetas, de forma tal que en las interrogantes se agrupen aquellas que permitan enriquecer la caracterización psicopedagógica del grupo. En Hablando de salud se pueden proponer acciones para conservar y mejorar su salud. En Vale la pena aparecen formas de enfrentamiento inadecuados que provoquen reflexión. En la tarjeta Entre tú y yo aparecerán refranes populares que provoquen reflexión sobre el tema y que les permita profundizar en él.

- Se jugará en pareja, cada jugador coloca en la salida una prenda que lo identifique, tira el dado y avanza tantas casillas como indique el número y de acuerdo con la casilla que le ha correspondido toma una tarjeta y responde.

- La reflexión y la socialización de la respuesta deben ser conducidas por el profesor.

- La meta es el símbolo de ganar pero la verdadera ganancia está en el clima y las posibilidades de comunicación que se creen en el grupo.

IV. Recomendaciones:

- .Adecuar las preguntas para poder instrumentar el juego en cualquier educación.

Fuente: Elaboración propia.

\section{Conclusiones}

- Los antecedentes abordados en el estudio indican que la etapa de la adolescencia constituye un período de cambios y no de crisis, si se aprovechan los diferentes contextos de actuación para ejercer influencia educativa a través de la comunicación, Si estos cambios no se tienen en cuenta, si los profesores y la familia no están preparados para enfrentarlos, se pueden producir contradicciones que repercuten negativamente en la formación de la personalidad de los adolescentes.

- La alternativa pedagógica propuesta propicia el trabajo educativo con los adolescentes, teniendo como punto de partida una acertada caracterización a través de la utilización novedosa de técnicas participativas. 


\section{Referencias}

Mercado Maldonado, A., \& Hernández Oliva, A. ( may./ago. de 2010). El proceso de construcción de la identidad colectiva. Convergencia, 17(53).

Ahumada, L. (2010). Distributed Leadership and Organizational Learning: Tensions and Contradictions of the Preferential School Subsidy Law in Rural Contexts. Psicoperspectivas, 111-123.

Almeida Macias, M., \& Ortiz Torre, E. (2016). Desarrollo de habilidades para la comunicación no verbal en la formacion inical del comunicador social. REFCalE: Revista Electrónica Formación y Calidad Educativa, 137-146.

Barbeta Viñas, M., \& Termes López, A. (2014). El rechazo escolar en diferentes contextos sociales: las resistencias y la reproducción de las contradicciones. Intersticios. Revista sociológica de pensamiento crítico.

Bautista Vallejo, J. M., \& López , N. (2002). El juego didáctico como estrategia de atención a la diversidad. Universidad de Huelva.

BONAL, X., ALEGRE, M., GONZÀLEZ, I., HERRERA, D., ROVIRA, M., \& SAURÍ, E. (2003). propiacions escolars. Usos i Sentits de l'Educació Obligatòria en l'Adolescència,.

García Hernández , T., Rodríguez Ferra , R., \& Castaño Hernández, S. (1999). Efectividad de las técnicas participativas en los conocimientos de adolescentes sobre enfermedades de transmisión sexual. Revista Cubana de Medicina General Integral, 536-540.

Izquierdo E., L. \&. (2015). Nuevos padres: construcción del rol paternal en hombres que participan activamente en la crianza de los hijos. Revista de investigaciones en Psicología , 33-55. doi: https://doi.org/10.15381/rinvp.v18i2.12082

Ojalvo Mitrany, V. (2002). La educación de valores. Reflexiones y experiencias desde el enfoque histórico-cultural. Tarbiya, Revista de Investigación e Innovación Educativa. Obtenido de https://revistas.uam.es/tarbiya/article/view/7390

Rodríguez Selpa, S. d., Socarrás Sánchez, S., Bujardón Mendoza, A., \& Iglesias Morel, N. (2015). Sistema de talleres con técnicas participativas para el fortalecimiento de la autodirección estudiantil. Humanidades Médicas, 511-530. 
Romero Pileta, I., Dopico Pérez, H. M., Fernández Téllez, I., Montoro Bombú, R., Chávez Cevallos, E., \& Contreras Calle, W. T. (2019). Análisis integral de la motivación en boxeadores. Revista Cubana de Investigaciones Biomédicas, 56-72.

Zaldívar, R. (2004). Técnicas Participativas Como Alternativa Para El Desarrollo De La Comunicación Oral Del Inglés. . Revista Pedagogía Universitaria .

\section{PARA CITAR EL ARTÍCULO INDEXADO.}

\section{Comas Benítez, M., Pérez Constante , M. B., Mora Avilés , B. E. \& Estupiñan Guamani, M. A. (2020).}

Adolescencia y Comunicación . ConcienciaDigital, 3(1.1), 444-455.

https://doi.org/10.33262/concienciadigital.v3i1.1.1175

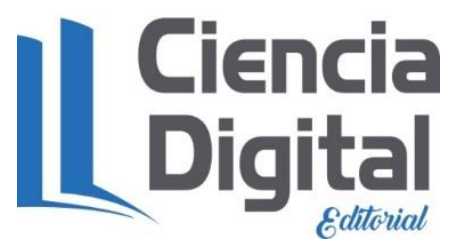

El artículo que se publica es de exclusiva responsabilidad de los autores y no necesariamente reflejan el pensamiento de la Revista Conciencia Digital.

El artículo queda en propiedad de la revista y, por tanto, su publicación parcial y/o total en otro medio tiene que ser autorizado por el director de la Revista Conciencia Digital.
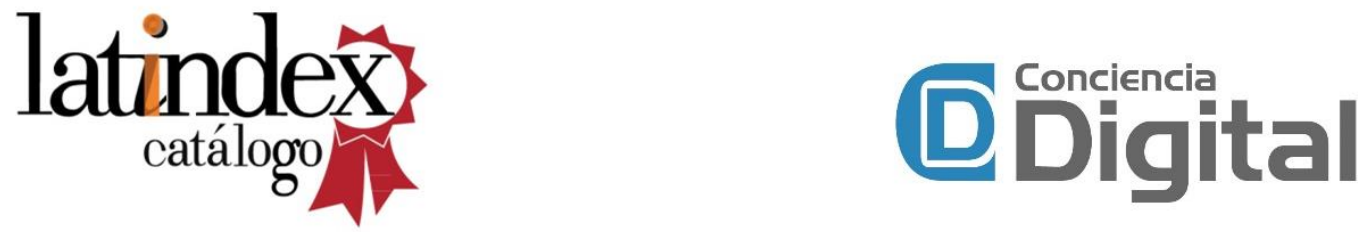